\title{
Efficiency of convection and Pre-Main Sequence lithium depletion
}

\author{
F. D’Antona ${ }^{1}$ and J. Montalbán ${ }^{2}$ \\ 1 INAF, Osservatorio Astronomico di Roma, 00040 Monteporzio, Italy \\ 2 Institut d'Astrophysique et de Géophysique, Université de Liège, 4000 Liège, Belgium
}

Received 21 February 2003 / Accepted 5 August 2003

\begin{abstract}
We show by detailed model computation how much the Pre-Main Sequence (PMS) lithium depletion depends on the treatment of over-adiabaticity, by taking advantage of the results of new models by Montalbán et al., which apply different treatments of convection to non-grey PMS models. In order to reproduce both the PMS lithium depletion (inferred from the lithium depletion patterns in young open clusters), and the location of PMS tracks in the HR diagram (inferred from the study of young PMS stars), convection both in the atmosphere and in a good fraction of the stellar envelope must be highly inefficient: e.g., in the Mixing Length Theory approximation, it must have a very low $\alpha=l / H_{\mathrm{p}}$. Unfortunately, the radii of these models are at variance with the solar radius, possibly indicating that there is some additional physical input, generally not taken into account in the stellar models, which affects the efficiency of convection in PMS stars, but probably not in the main sequence stars nor in evolved red giants. We stress the importance of determining precisely masses and lithium abundance in PMS binaries such as the important spectroscopic and eclipsing binary RXJ 0529.4+0041.
\end{abstract}

Key words. stars: evolution - stars: atmospheres - convection - stars: pre main sequence

\section{Introduction}

The location in the HR diagram of the Pre-Main Sequence (PMS) evolutionary tracks is very sensitive to physical inputs such as low-temperature opacity, equation of state, rotation, atmosphere model, and the treatment of convection. During the last years, much work has been done to improve the knowledge of low-temperature opacities and to include them in the modeling of stellar atmospheres. Among the many theoretical evolutionary tracks today available (new convection treatment with grey boundary conditions; classic convection with non-grey atmosphere models...) it is difficult to disentangle the effect of the different physical inputs on the results. In Paper I (Montalbán et al. 2003) we made a detailed exploration of models, with the aim to extricate the different roles of non-grey atmospheres and of convection in the track computations. This task has been approached by comparing a large number of models, having different assumptions for the treatment of convection both in the atmosphere and in the interior, and using different grids of atmospheric computations. In particular, we have shown that a stellar model is fully described only when we specify not only the atmospheric model used as boundary condition to the interior, but also the convection parameters used for the

Send offprint requests to: F. D'Antona, e-mail: dantona@coma.mporzio.astro.it atmospheric grid, and the value of optical depth at which the boundary conditions are taken, that is the photospheric "matching point" $\tau_{\mathrm{ph}}$. In fact, until now, the "expensive" model atmosphere computations have been generally performed only for one specific convection model, e.g. a given ratio of mixing length to pressure scale heigth, in the Mixing Length Theory (MLT), $\alpha=\alpha_{\mathrm{atm}}$. The value of $\alpha$ can be changed only in the computation of the interior $\left(\alpha=\alpha_{\text {in }}\right)$ e.g. to fit the solar radius in the solar model. However, if a large value of $\tau_{\mathrm{ph}}$ is chosen as matching point between the atmosphere and the interior, the most superadiabatic part of the convection zone is all included in the atmosphere. Consequently, the changing of $\alpha_{\text {in }}$ does not affect the model in the same way as a full change the convection parameter in the whole model, including the atmosphere (see Paper I).

Although this may seem only of academic interest, it is of subtle importance, if we wish to progress in one of the most intriguing problems of the stellar structure, namely the problem of PMS lithium depletion. In fact, the well known "problem of lithium in the Sun", which in the years 1965-1990 was mainly that solar models could not burn any substantial fraction of their initial lithium during the PMS, was generally taken as a good proof that additional mechanisms for depletion were required, acting during the long solar MS lifetime. This interpretation is today taken as the most plausible one, confirmed by the variation of the Lithium Depletion Patterns (LDP) in open clusters 
(see e.g. Chaboyer 1998). In fact the lithium vs. $T_{\text {eff }}$ relation for the MS stars of young open clusters indicates a lithium depletion by at most a factor two for the solar mass in young clusters, while it is compatible with the solar depletion (a factor $\sim 140$ with respect to the solar system abundance) in some stars of the cluster M 67, close to the solar age. For recent reviews see e.g. Jeffries (2000) and Pasquini (2000).

However, a different problem emerges from the most recent computation of solar models: they deplete too much lithium during the PMS evolution (D’Antona \& Mazzitelli 1994, 1997; Schlattl \& Weiss 1999; Piau \& Turck-Chièze 2002) and are incompatible with the open clusters observations ${ }^{1}$. This problem is most severe in models using very efficient convection models, in fact it is more relevant for the D'Antona \& Mazzitelli (1994, 1997) models adopting the Full Spectrum Turbulence (FST) convection by Canuto \& Mazzitelli (1991) and/or by Canuto et al. (1996). MLT models of the most recent generation, adopting updated equations of state and opacities also deplete too much lithium: notice however that the problem is found mainly in tracks whose convection is adjusted to provide the solar radius at the solar age! If one does not require the solar fit, it is easy to decrease the convection efficiency and to obtain a small PMS lithium depletion.

A similar problem is found when comparing the location of PMS theoretical tracks with the observed few data of PMS stars for which an independent determination of mass is available, either because they belong to binaries (e.g. Covino et al. 2001; Steffen et al. 2001) or by the measure of the dynamical properties of their protoplanetary disks (Simon et al. 2000): the tracks most consistent with the observations are those with cooler atmospheres (higher mass for a given spectral type) and thus those which, generally, provide a radius larger than $R_{\odot}$ for the solar model. In particular, we can quote the analysis by Simon et al. (2000): their results are consistent, - within the statistical errors - with the set of tracks whose evolution for the solar mass does not pass through the solar location. In addition, their observations are not consistent with the FST tracks. In fact, for any value of its fine tuning parameters, the FST convection always provides solar tracks which pass within $\sim 100 \mathrm{~K}$ of the solar location.

These two problems, HR diagram location of the tracks during the PMS evolution, and lithium depletion, are necessarily correlated each other, because, the smaller the $T_{\text {eff }}$ of the Hayashi track, the smaller will be the temperature at the base of the convective envelope during the possible phase of lithium burning (see, e.g. D'Antona et al. 2000). Having computed several sets of stellar models, which are fully described in Paper I, we decided to look at the lithium depletion with different assumptions. In Sect. 2 we show and discuss the results. In Sect. 3 we show that the models having low lithium depletion also reproduce better the location of PMS stars in the HR diagram, but they do not reproduce the solar radius. Thus the problem is now better defined: the efficiency of convection in PMS must be highly reduced with respect to the efficiency in MS.

\footnotetext{
1 We mention, as a caveat, that these models are not strictly incompatible with the present solar lithium abundance, if we accept that all or most of the solar lithium was depleted at the PMS stage.
}

In particular, those tracks whose convection formulation can not provide a solar model with radius far different from the solar radius - noticeably the FST models - can not reproduce the location in PMS and its lithium depletion, unless the efficiency of their convective transport is altered by another physical input acting during the PMS, but not during the MS or post-MS giant phase.

\section{Standard models: Fitting the solar radius, or not}

Our models are computed following standard Henyey type integration, by means of the ATON2.0 code (Ventura et al. 1998a; Montalbán et al. 2001) updated in Paper I to include as surface boundary conditions (BCs) the new grids of ATLAS9 atmospheres from Heiter et al. (2002a). We consider here the models having solar metal abundance in mass fraction $Z=0.02$ and helium abundance $Y=0.28$. The initial lithium abundance was taken as $\log N(\mathrm{Li})=3.31 \pm 0.04$, the solar system abundance given by Anders \& Grevesse (1989). The PMS lithium burning starts when the temperature reaches $\sim 2 \times 10^{6} \mathrm{~K}$ during the PMS contraction. The important inputs are the temperature and density at the bottom of the convective region. Actually lithium burning generally begins approximately at the bottom of the Hayashi track, when a radiative core develops and grows during the evolution towards the main sequence. Figure 1 shows the the central density, the density at the bottom of the convective region, and the surface lithium abundance along two tracks of $1 M_{\odot}$, whose atmosphere is interpolated in the grid of models by Allard \& Haushildt (1997). Varying the convection treatment in the interior from $\alpha_{\text {in }}=l / H_{\mathrm{p}}=1$ to 1.9 , increases the temperature at the bottom of the envelope by $\sim 3 \%$. As we see, the density is instead increased by $\sim 50 \%$. The difference in the structure is sufficient to change the lithium depletion by a factor $\sim 10$. As shown in Fig. 1, the lithium burning phase is completed when the age is still as low as $10^{7} \mathrm{yr}$. These models do not include mechanisms for depletion other than plain convective mixing. These latter, however, act on a much longer timescale during the MS phase, so that the remaining lithium abundance should be preserved at least until the age of the Pleiades (see, e.g. Pinsonneault et al. 1990). Notice that lithium burning begins approximately when the radiative core develops (in Fig. 1, where the central density curve detaches from the density at the bottom of convection). This occurs at the knee in the HR diagram, where the track shifts from its convective behavior (the Hayashi track) to the so called Henyey - radiative - line of approach to the MS (e.g. Stahler 1988). Thus the lithium burning begins at a $T_{\text {eff }}$ around the minimum luminosity of the track, which we tentatively use as a possible interesting parameter. This $T_{\text {eff-lim }}$ gives us an indication of the PMS track location in the HR diagram (being smaller for a smaller efficiency of convection) and we expect that it might be correlated with the PMS lithium burning.

Table 1 shows the remaining lithium abundance in the solar tracks after the PMS depletion, in several models from Paper I, all of which fulfilling the requirement that the solar radius is achieved at the solar age. The models are either computed by using the new Vienna ATLAS9 (Kurucz 1993, 1995, 1998) model atmospheres (Heiter et al. 2002) or the NextGen 


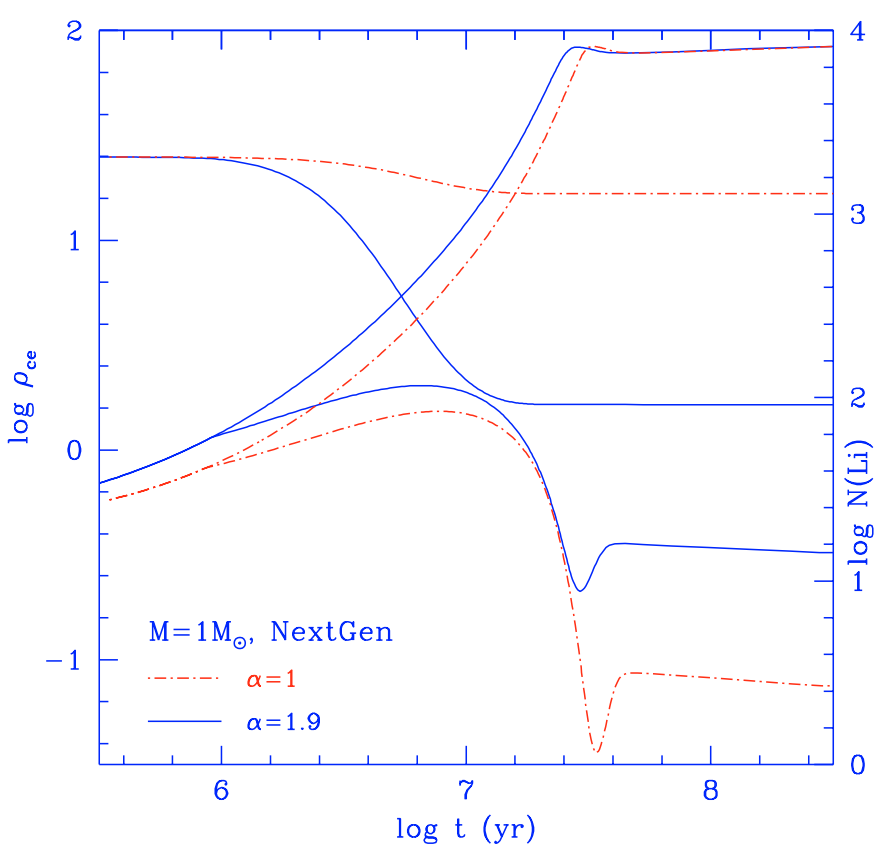

Fig. 1. Along two tracks for the solar mass, differing in $\alpha_{\text {in }}$, we show the lithium depletion (upper curves, scale on the right) and the central density and the density at the bottom of the convective envelope (curves which detach at $\log t \sim 6$, scale on the left).

Table 1. Lithium remnant after the PMS depletion in $1 M_{\odot}$ models, starting with $\log N(\mathrm{Li})_{\text {in }}=3.31$.

\begin{tabular}{lcccll}
\hline \hline atmosphere & $\alpha_{\text {atm }}$ & interior & $\alpha_{\text {in }}$ & $\tau_{\mathrm{ph}}$ & $\log N(\mathrm{Li})$ \\
\hline ATLAS9 MLT & 0.5 & MLT & 1.85 & 3 & 1.864 \\
ATLAS9 MLT & 0.5 & MLT & 2.3 & 100 & 2.130 \\
ATLAS9 MLT & 0.5 & MLT & 6.3 & 10 & 2.564 \\
ATLAS9 FST & 0.1 & FST & 0.2 & 10 & 1.537 \\
AH97 MLT & 1.0 & MLT & 1.9 & 3 & 1.960 \\
AH97 MLT & 1.0 & MLT & 1.9 & 100 & 2.62 \\
\hline
\end{tabular}

models by Allard \& Hauschildt (1997, AH97). Among the ATLAS9 models, some are computed by the Full Spectrum Turbulence (FST) convection description by Canuto et al. (1996) both in the atmosphere and in the interior. We see that depletion is very severe in the FST model. For the MLT models computed with the Vienna ATLAS9 model atmospheres (Heiter et al. 2002) which have $\alpha_{\text {atm }}=0.5$, the solar fit is achieved by assuming a larger $\alpha_{\text {in }}$ in the interior computation. The larger the $\tau_{\mathrm{ph}}$ at which the atmosphere ends, the larger is the $\alpha_{\text {in }}$ required for the solar fit, and the smaller the lithium depletion. In fact, the larger is $\tau_{\mathrm{ph}}$, the larger is the fraction of the over-adiabatic region integrated using a low efficiency of convection, so that the $T_{\text {eff }}$ of the model is significantly reduced, as well as the temperature at the bottom of the convective envelope at the epoch of lithium burning. Nevertheless, the minimum depletion achieved in these models is $\sim 0.6 \mathrm{dex}$, still too much to be compatible with the open cluster LDPs (see later). Thus we confirm that these models using non-grey boundary conditions have a strong lithium depletion, as it is obtained in the grey MLT models (e.g. Schlattl \& Weiss 1999;
Table 2. Lithium depletion in NextGen based models with $\alpha=1$ and $\alpha=1.9$ in the interior.

\begin{tabular}{cccll}
\hline \hline$M / M_{\odot}$ & $\alpha_{\text {in }}$ & $\tau_{\text {ph }}$ & $\log T_{\text {eff-lim }}$ & $\log N(\mathrm{Li})$ \\
\hline 1.1 & 1.9 & 3 & 3.663 & 2.93 \\
1.1 & 1.9 & 10 & 3.653 & 2.93 \\
1.1 & 1.9 & 100 & 3.650 & 2.93 \\
1.1 & 1 & 3 & 3.619 & 3.27 \\
1.1 & 1 & 10 & 3.615 & 3.27 \\
1.1 & 1 & 100 & 3.613 & 3.27 \\
1 & 1.9 & 3 & 3.649 & 1.96 \\
1 & 1.9 & 10 & 3.637 & 2.52 \\
1 & 1.9 & 100 & 3.633 & 2.62 \\
1 & 1.0 & 3 & 3.604 & 3.111 \\
1 & 1.0 & 10 & 3.609 & 3.249 \\
1 & 1.0 & 100 & 3.607 & 3.236 \\
0.9 & 1.9 & 3 & 3.631 & 0.845 \\
0.9 & 1.9 & 10 & 3.619 & 1.920 \\
0.9 & 1.9 & 100 & 3.615 & 2.118 \\
0.9 & 1.0 & 3 & 3.597 & 2.880 \\
0.9 & 1.0 & 10 & 3.593 & 2.980 \\
0.9 & 1.0 & 100 & 3.595 & 2.930 \\
0.8 & 1.9 & 3 & 3.604 & 0.85 \\
0.8 & 1.9 & 10 & 3.609 & 1.93 \\
0.8 & 1.9 & 100 & 3.607 & 2.12 \\
0.8 & 1.0 & 3 & 3.582 & 2.345 \\
0.7 & 1.9 & 3 & 3.604 & -3.36 \\
0.7 & 1.9 & 100 & 3.609 & -1.49 \\
\hline & & & &
\end{tabular}

Piau \& Turck-Chièze 2002) as well as in the grey FST models (D’Antona \& Mazzitelli 1994, 1997).

Table 2 shows the lithium depletion for masses from $1.1 M_{\odot}$ to $0.7 M_{\odot}$ in two sets of MLT models computed using Allard \& Hauschildt (1997) NextGen model atmospheres as boundary condition. All these models consider $\alpha_{\mathrm{atm}}=1$, the value fixed in the grid of model atmospheres, but one set is computed assuming $\alpha_{\text {in }}=1.9$ in the interior, so that the solar track fits the solar radius, and in the other set we adopt the same value than in the atmosphere, $\alpha_{\text {in }}=1$, even if the corresponding evolutionary track does not fit the Sun. These latter models, whichever is the matching point $\tau_{\mathrm{ph}}$, have very similar lithium depletion ${ }^{2}$.

In Table 2 we also list the parameter $T_{\text {eff-lim, and Fig. } 2}$ shows the lithium depletion versus $T_{\text {eff-lim: for large enough }}$ masses $\left(M \gtrsim 1.1 M_{\odot}\right)$ the depletion is small for any convection efficiency, but the $T_{\text {eff }}$ location of the track increases with the convection efficiency ( $T_{\text {eff-lim }}$ increases). For the lowest masses $\left(0.7 M_{\odot}\right)$ the $T_{\text {eff }}$ location does not vary significantly, but the

\footnotetext{
${ }^{2}$ If the interior and atmospheric physics were exactly the same, we should obtain the same model structure, at least for the $\tau_{\mathrm{ph}}=10$ and 100 models (at $\tau_{\mathrm{ph}}=3$ the diffusion approximation is not yet strictly valid and this may give some differences). Actually, also numerical differences can be responsible for some variation in the results: in fact the atmospheric structure in the AH97 model atmospheres includes only very few mesh points between $\tau=10$ and 100, while the interior integration is much more detailed.
} 


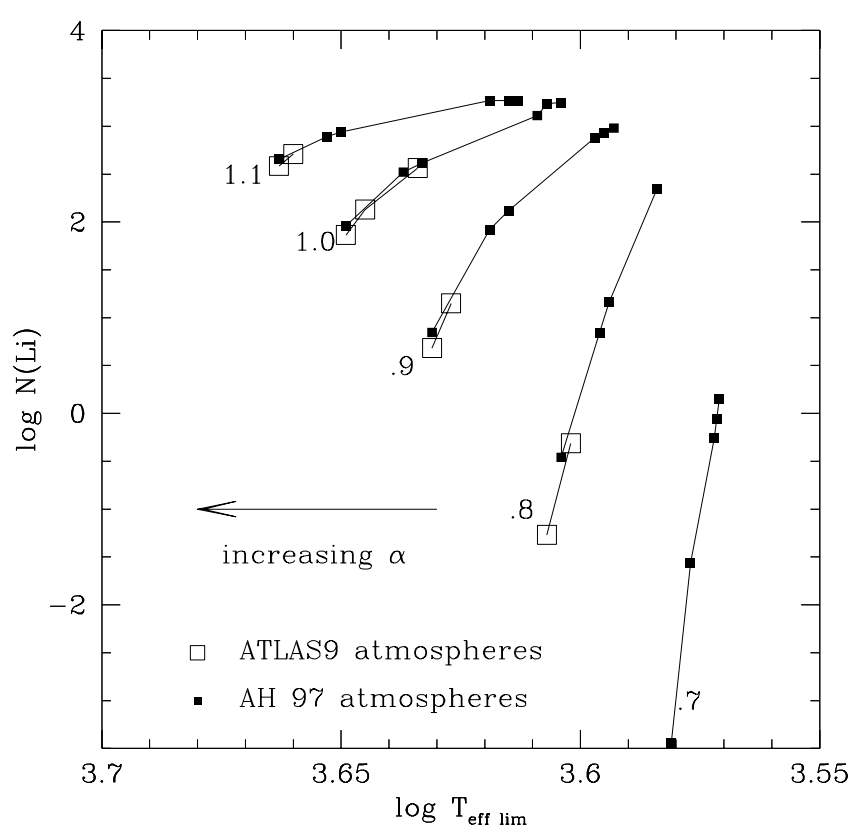

Fig. 2. Lithium versus $T_{\text {eff-lim }}$ relation for tracks from 1.1 to $0.7 M_{\odot}$, as labeled in the figure. The full dots are the models computed with AH97 model atmospheres, while the open squares employ the ATLAS9 MLT Vienna model atmospheres by Heiter et al. (2002). The $0.8 M_{\odot}$ models of $\alpha_{\text {atm }}=\alpha_{\text {in }}=1$ coincide at the top of the sequence of $0.8 M_{\odot}$ models, while they differ slightly for the other masses, due to possible differences in the input physics of the model atmosphere and interior.

lithium depletion varies by several orders of magnitude from the models with low to those with high convection efficiency. The masses $0.8-1 M_{\odot}$ show a wider range of variability, and a very significant degree of correlation between $T_{\text {eff-lim }}$ and the lithium depletion.

Figure 2 also shows the points corresponding to the ATLAS9 MLT models fitting the Sun: these have very similar lithium depletion, for the solar track, to that of the AH97 models also fitting the Sun. For the other masses, the results differ, but they follow the same pattern along the proper relation lithium $-T_{\text {eff-lim. }}$. We did not consider ATLAS9 models not fitting the Sun, but, if we had assumed such a low efficiency of convection also in the interior, we would have obtained smaller lithium depletions, as shown by the AH97 models with $\alpha=1$ both in the atmosphere and in the interior. We immediately recognize that the lithium depletion in young open clusters requires the minimum efficiency of convection among those we have considered: only the models with AH97 model atmospheres and $\alpha_{\text {atm }}=\alpha_{\text {in }}=1$ can fit the open cluster lithium data, although the tracks do not fit the solar location. This is shown in Fig. 3 where we compare the lithium depletion predicted by the models in Table 2 with the Pleiades data by Soderblom et al. (1993) \& Garcia Lopez et al. (1994). We have normalized the lithium abundances in the models to an initial abundance $\log N(\mathrm{Li})=3.1$. We see that only upper squares, corresponding to the models with $\alpha_{\text {atm }}=\alpha_{\text {in }}=1$, are compatible with the data. In addition, all the models of $0.7 M_{\odot}$ have depletion larger than allowed by the data, suggesting that some additional phenomenon is taking place at lower $T_{\text {eff }}$. In the

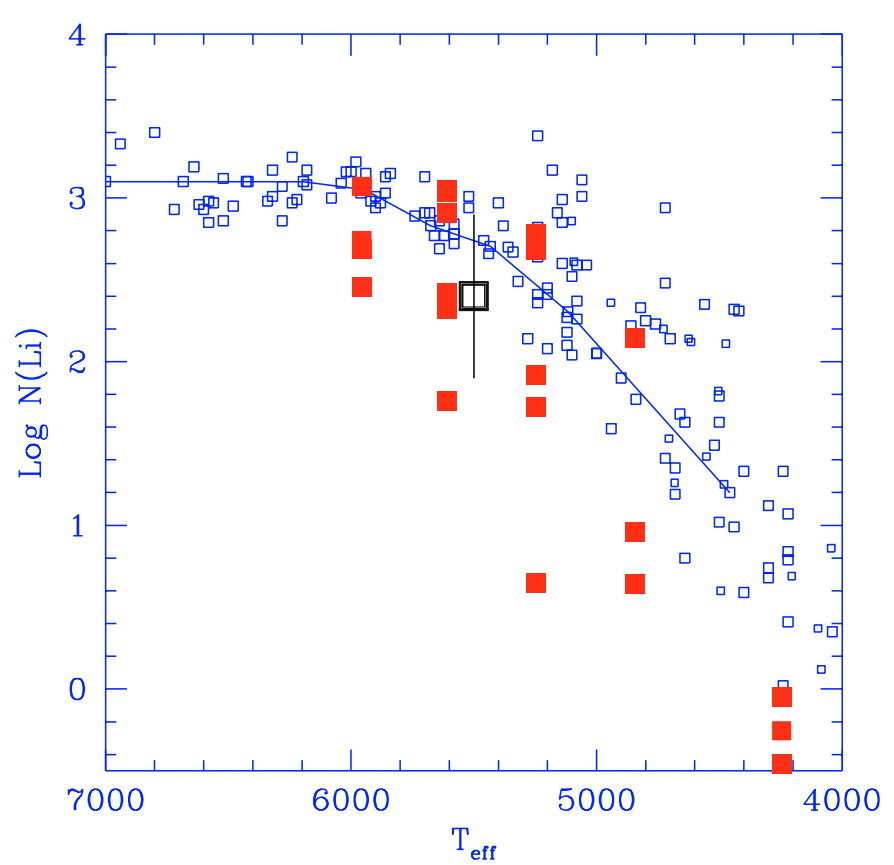

Fig. 3. The Pleiades data by Soderblom et al. (1993) and Garcia Lopez et al. (1994) (open squares) are compared with the depletion predicted by the models in Table 2 (full big squares). The models are placed at the $T_{\text {eff }}$ they would take in an empirical MS, at the Pleiades age. Only the upper squares, corresponding to the models with $\alpha_{\text {atm }}=\alpha_{\text {in }}=1$, are compatible with the data. The full line shows the depletion from the models by Ventura et al. (1998b) computed including the thermal effect of a magnetic field on the convective temperature gradients. The large open square with the error bar represents the lithium abundance of the secondary component of RXJ 0529.4+0041 $(\log N(\mathrm{Li})=2.4 \pm$ 0.5 , Covino et al. 2001). The $T_{\text {eff }}$ at which the point is located $(5500 \mathrm{~K})$ is assumed to be the main sequence $T_{\text {eff }}$ of a star of mass $0.925 M_{\odot}$.

figure we also plot (full line) the depletion from the models by Ventura et al. (1998b) computed including the thermal effect of a dynamo induced magnetic field on the convective temperature gradients. In this latter case, the magnetic field acts to reduce the convective efficiency, allowing the fit with the cluster LDP. We conclude that PMS depletion is compatible only with models in which PMS convection is much less efficient than MS convection.

\section{Constraints on the PMS location of tracks}

We have mentioned that the (scarce) data on PMS masses seem to indicate that the tracks most compatible, in terms of $T_{\text {eff }}$ and luminosity, with the observations are those in which convection is less efficient (Covino et al. 2001; Steffen et al. 2001; Simon et al. 2000). A valid addition to the analysis can be the consideration of the lithium content. An example of how the analysis could be done is shown by means of the only relevant observation available, concerning the secondary of the eclipsing double-lined spectroscopic binary, containing both components in PMS, RXJ 0529.4+0041 (Covino et al. 2000). This system provides powerful constraints: it is a very important indicator of the fundamental stellar parameters, as masses and radii of the components are simultaneously derived. Figure 3 shows the location of the secondary of RXJ 0529.4+0041 in the 

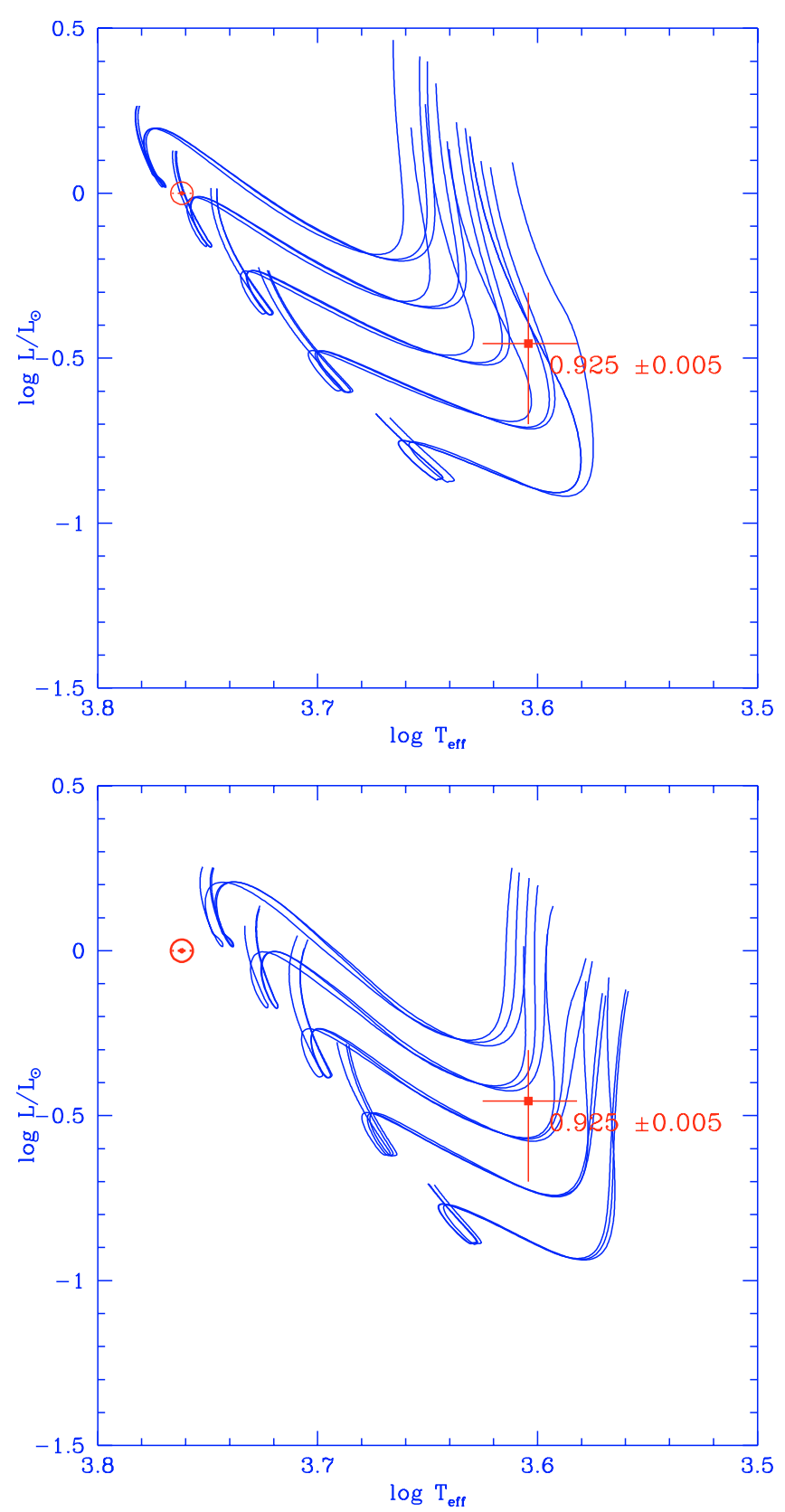

Fig. 4. The top figure shows the tracks computed with AH97 atmospheres and $\alpha_{\text {in }}=1.9$, while the bottom figure shows the tracks with $\alpha_{\text {in }}=1.0$. For each mass $\left(1.1,1.0,0.9,0.8\right.$ and $0.7 M_{\odot}$, from top to bottom) the three curves represent the tracks obtained for different $\tau_{\mathrm{ph}}$. The solar location is shown: it is compatible with the solar model having $\alpha_{\text {in }}=1.9$, but $\sim 400 \mathrm{~K}$ hotter than the solar model with $\alpha_{\text {in }}=1.0$. The location of the secondary component of the binary RXJ 0529.4+0041 is also shown.

HR diagram, compared with the tracks having convection parameters fitting the Sun (upper panel) or not fitting the Sun (lower panel). The mass of this star is given as $0.925 \pm 0.005 M_{\odot}$ in Covino et al. (2001). We see that both sets of tracks are compatible with this mass within the errors. The tracks of 1 and $0.9 M_{\odot}$ in the sets not fitting the Sun very nicely bracket the star location and seem most adequate. Notice that in the following we rely on the assumption that both the Covino et al. (2001) data and the results concerning the HR diagram location of PMS objects can be taken at face value. Actually, the errors on the $T_{\text {eff }}$ determination might result to be larger than given by the authors, and for this reason we judge that a more quantitative analysis is still premature. The lithium abundance determination by Covino et al. (2000), $\log N(\mathrm{Li})=$ $2.4 \pm 0.5$ is shown in Fig. 3. As the star may still be at a phase in which PMS depletion is going on, this determination is an upper limit to the future location of the star in the [lithium vs. MS $\left.-T_{\text {eff }}\right]$ plane. According to the authors, actually the abundance determination should be regarded as a lower limit, as it was done by using the Pavlenko \& Magazzù (1996) model atmospheres for a gravity $\log g=4.5$, more appropriate for main sequence gravities. Reduction of the gravity would thus provide a larger abundance. In spite of its large error, this value, considered as a lower limit, is therefore well compatible with the quite low depletion of the $\alpha_{\text {in }}=1$ models and with the average lithium depletion expected from the observations in young clusters. Further studies of this system, and in particular a better determination of its lithium abundance, are important to help us in discriminating how inef ficient convection must be to be compatible with the se data.

\section{Conclusions}

Although the parametrizations used for convection are highly unsatisfactory, we have shown that there are two different independent indications which imply that convection in the PMS phase must be highly inefficient:

1. the location in the HR diagram of the few objects for which the mass (or an upper value of the mass) is known, according to several comparisons appearing in the most recent astronomical literature (although we have still to remind that further work is necessary to understand carefully the $T_{\text {eff }}$ scale of PMS objects).

2. the PMS depletion of lithium, according to the well known observations of LDPs in young open clusters, plus the determination of the lithium abundance in the secondary component of the system RXJ 0529.4+0041, whose mass is known with high precision.

We have also shown that the PMS location of the tracks in the HR diagram and the corresponding lithium depletion during the PMS stage are correlated, for those masses in which lithium depletion is significant but not complete. Any effort to increase our knowledge of the PMS masses and/or of the lithium content in young stars will add further weight to our main conclusion, which is that we can not parametrize the efficiency of convection in the same way in the MS and in the PMS. A potential implication of this result, preliminary explored in some previous work (Ventura et al. 1998b; D’Antona et al. 2000) is that there might be an additional parameter playing a role in this game, connected with the fast rotation of the PMS stars.

Acknowledgements. We acknowledge support from the Italian Space Agency ASI under the contract ASI I/R/037/01. J.M. also acknowledge the support of Osservatorio Astronomico di Roma. 


\section{References}

Alexander, D. R., \& Ferguson, J. W. 1994, ApJ, 437, 879

Allard, F., \& Hauschildt, P. 1997 (AH97) NextGen http://dilbert.physast.uga.edu/ yeti/mdwarfs.html

Anders, E., \& Grevesse, N. 1989, Geochim. Cosmochim. Acta, 53, 197

Böhm-Vitense, E. 1958, Z. Astrophys., 46, 108

Canuto, V. M., Goldman, I., \& Mazzitelli, I. 1996, ApJ, 473, 550

Chaboyer, B. 1998, in New eyes to see inside the sun and stars, ed. F.-L. Deubner, J. Christensen-Dalsgaard, \& D. Kurtz, IAU Symp., 185,25

Covino, E., Melo, C., Alcalá, J. M., et al. 2001, A\&A, 375, 130

D'Antona, F. 2000, Star formation from the small to the large scale, ESLAB Symp. (33:1999: Noordwijk, The Netherlands), ed. F. Favata, A. Kaas, \& A. Wilson, Proc. of the 33rd ESLAB Symp. on star formation from the small to the large scale, ESTEC, Noordwijk, The Netherlands, 2-5 November 1999 Noordwijk, The Netherlands: European Space Agency (ESA), 2000, ESA SP, 445,161

D’Antona, F., \& Mazzitelli, I. 1994, ApJS, 90, 467 (DM94)

D'Antona, F., \& Mazzitelli, I. 1997, in Cool Stars in Clusters and Associations, ed. G. Micela, \& R. Pallavicini, Mem. S.A.It. 68, 807

D'Antona, F., Ventura, P., \& Mazzitelli, I. 2000, ApJL, 543, L77

D’Antona, F., Montalbán, J., Kupka, F., \& Heiter, U. 2002, ApJ, 564, L93

Garcia Lopez, R. J., Rebolo, R., \& Martin, E. L. 1994, A\&A, 282, 518

Heiter, U., Kupka, F., van't Veer-Menneret, C., et al. 2002a, A\&A, 392,619
Jeffries, R. D. 2000, Stellar Clusters and Associations: Convection, Rotation, and Dynamos, ASP Conf. Ser., 198, 24

Kurucz, R. L. 1998, http: //cfaku5.hardvard.edu/

Kurucz, R. L. 1995, CD-ROM No 13, revised

Kurucz, R. L. 1993, ATLAS9 Stellar Atmosphere Programs and $2 \mathrm{~km} / \mathrm{s}$ grid (Kurucz CD-ROM No 13)

Mazzitelli, I., \& Moretti, M. 1980, ApJ, 235, 955

Montalbán, J., Kupka, F., D’Antona, F., \& Schmidt, W. 2001, A\&A, 370, 982

Montalbán, J., D’Antona, F., Kupka, F., \& Heiter, U. 2003, A\&A, submitted (Paper I)

Pavlenko, Y. V., \& Magazzu, A. 1996, A\&A, 311, 961

Pasquini, L. 2000, IAU Symp., 198, 269

Piau, L., \& Turck-Chièze, S. 2002, 566, 419

Pinsonneault, M. H., Kawaler, S. D., \& Demarque, P. 1990, ApJS, 74, 501

Schlattl, H., \& Weiss, A. 1999, A\&A, 347, 272

Simon, M., Dutrey, A., \& Guilloteau, S. 2000, ApJ, 545, 1034

Soderblom, D. R., Jones, B. F., Balachandran, S., et al. 1993, AJ, 106, 1059

Stahler, S. W. 1988, PASP, 100, 1474

Steffen, A. T., Mathieu, R. D., Lattanzi, M. G., et al. 2001, AJ, 122, 997

Ventura, P., Zeppieri, A., Mazzitelli, I., \& D’Antona, F. 1998a, A\&A, 334,953

Ventura, P., Zeppieri, A., Mazzitelli, I., \& D’Antona, F. 1998b, A\&A, 331,1011 